\title{
Second Trimester Spontaneous Abortion
}

National Cancer Institute

\section{Source}

National Cancer Institute. Second Trimester Spontaneous Abortion. NCI Thesaurus.

Code C128780.

Pregnancy loss between 14 weeks 0 days and 21 weeks 6 days gestation. 